\title{
Kants Hand, Chiralität und konvexe Polytope
}

Karl Wirth und André S. Dreiding

Karl Wirth studierte an der ETH Zürich Mathematik, promovierte bei M. Jeger und arbeitete mit A.S. Dreiding zusammen. Danach war er als Mathematiklehrer an der Kantonalen Maturitätsschule für Erwachsene in Zürich tätig. Seit seiner Pensionierung beschäftigt er sich wieder vermehrt mit Themen der mathematischen Chemie.

André S. Dreiding promovierte an der Universität Michigan und ist Professor emeritus für Organische Chemie an der Universität Zürich. Mit dem Ziel, geometrische Modellvorstellungen von Molekülen zu systematisieren, startete er 1970 ein interdisziplinäres Forschungsprojekt unter Mitwirkung von Informatikern und Mathematikern.

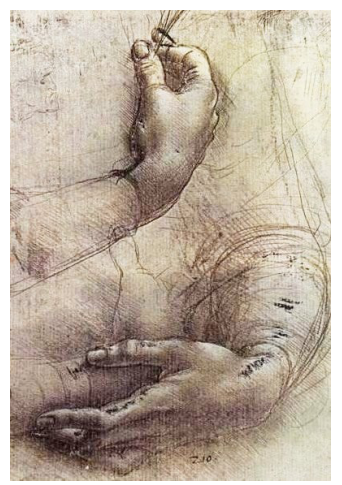

manche meinen lechts und rinks kann man nicht velwechsern, werch ein illtum!

Ernst Jandl

Worin unterscheidet sich eine rechte von einer linken Hand, trifft doch jede Eigenschaft der einen Hand auch auf die andere zu? Diese von Kant gestellte Frage und die von ihm gegebene Antwort waren immer wieder Anlass zu Kontroversen über die Natur des Raumes. Einen klärenden Beitrag lieferte der Mathematiker Reidemeister, dessen Aussagen in dieser Arbeit unter Verwendung eines in der Chemie wichtigen Begriffs interpretiert werden, nämlich der Chiralität. Im Weiteren geht es um die Tragweite des aus dieser Deutung hervorgehenden Phänomens der Diachiralität und um das Bilden von Chiralitätsklassen chiraler Objekte durch Orientierung. In einem zweiten Teil werden die besprochenen Chiralitätsaspekte auf konvexe Polytope angewendet, wobei u.a. ein Minimierungsverfahren und ein darauf basierender Algorithmus wesentlich sind. 


\title{
1 Kants Paradoxon
}

Immanuel Kant (1724-1804) gilt als der grosse Philosoph, der sich mit den Möglichkeiten und Grenzen der menschlichen Vernunft befasste. Viel ist im Kant-Jahr 2004, in dem sich sein Todestag zum zweihundertsten Mal jährte, über sein philosophisches Werk geschrieben worden. Nach wie vor wenig bekannt sind seine mehr mathematisch-naturwissenschaftlichen Schriften, die vor allem aus der frühen Schaffensperiode stammen. So beschäftigte er sich in einer Abhandlung mit dem Titel Von dem ersten Grunde des Unterschiedes der Gegenden im Raum [6] aus dem Jahre 1768 mit einem Phänomen, das in den modernen Naturwissenschaften eine herausragende Rolle spielt und heute mit dem Begriff Chiralität (griech. cheir $=$ Hand) umschrieben wird. In dieser Abhandlung ist zu lesen:

\begin{abstract}
„Die rechte Hand ist der linken ähnlich und gleich, und, wenn man bloß auf eine derselben allein sieht, auf die Proportion und Lage der Theile unter einander, und auf die Größe des Ganzen, so muß eine vollständige Beschreibung der einen, in allen Stücken auch von der anderen gelten. (...)

Weil aber gar kein Unterschied in dem Verhältnisse der Theile derselben unter sich Statt findet, sie mag eine Rechte oder Linke seyn, so würde diese Hand in Ansehung einer solchen Eigenschaft gänzlich unbestimmt seyn, d.i. sie würde auf jede Seite des menschlichen Körpers passen, welches unmöglich ist.“
\end{abstract}

Kant stellt fest, dass zwei als verschieden empfundene Objekte, etwa eine rechte und eine linke Hand, (idealisiert) in ihren metrischen Eigenschaften vollständig übereinstimmen können: Jeder bei der einen Hand gemessene Abstand kann entsprechend auch bei der andern gemessen werden. Heute sagt man, die beiden Hände seien isometrisch. Kant formuliert dann ein Paradoxon: Weil die beiden Hände isometrisch sind, müsste jede zu beiden handlosen Körperhälften passen, die (idealisiert) ebenfalls als isometrische Objekte anzusehen sind. Dies würde aber der Erfahrung widersprechen, wonach eine Hand nur zu einer Körperhälfte passt. Wie hat nun Kant versucht, diesen Widerspruch aufzuklären? Wir zitieren:

\begin{abstract}
„Es ist hieraus klar: daß nicht die Bestimmungen des Raumes Folgen von den Lagen der Theile der Materie gegen einander, sondern diese Folgen von jenen seyn, und daß also in der Beschaffenheit der Körper Unterschiede angetroffen werden können, und zwar wahre Unterschiede, die sich lediglich auf den absoluten und ursprünglichen Raum beziehen; weil nur durch ihn das Verhältniß körperlicher Dinge möglich ist, und daß, weil der absolute Raum kein Gegenstand einer äußeren Empfindung, sondern ein Grundbegriff ist, der alle dieselbe zuerst möglich macht, wir dasjenige, was in der Gestalt eines Körpers lediglich die Beziehung auf den reinen Raum angehet, nur durch die Gegenhaltung mit anderen Körpern vernehmen können.“
\end{abstract}

Dieser schwer lesbare Text beinhaltet nach unserem Verständnis, dass es für den Unterschied isometrischer Objekte von der Art unserer beiden Hände keinen in den Objekten selbst liegenden Grund geben könne. Dieser müsse deshalb ausserhalb der Objekte zu finden sein. Kant schreibt diesen Unterschied einer Raumeigenschaft zu, die sich nicht auf 
die gegenseitige Lage der Teile, d.h. auf Abstände, zurückführen lässt. Er bezeichnet den Raum mit dieser Eigenschaft als ,absolut'; in Bezug zu ihm seien eine rechte und eine linke Hand unterschiedlich. Damit stellt sich Kant auf die Seite Newtons, der ein gutes halbes Jahrhundert früher, in einem Disput mit Leibniz, ebenfalls einen absoluten Raum mit ewig fixen Orten postulierte. Nach Leibniz'scher Auffassung hingegen können Orte erst durch Bezüge festgelegt sein.

Der Standpunkt von Leibniz setzte sich nach und nach durch, und die eher religiös beeinflussten Vorstellungen vom absoluten Raum wichen bis zum zwanzigsten Jahrhundert zunehmend einer logisch-mathematischen Argumentation. Verschiedentlich haben sich Mathematiker mit der Kant'schen Problematik beschäftigt. Es war dann aber vor allem K. Reidemeister, der sich ausführlich mit Kants Paradoxon auseinandersetzte und Argumente gegen seine Schlussfolgerungen lieferte. Er legte sie in seinem 1957 veröffentlichten Buch Raum und Zeit [9] dar. Reidemeister lehrte 1925-1933 im ,Kant'schen “ Königsberg, wurde 1934 nach Marburg berufen und war ab 1955 in Göttingen tätig. Seine Forschungen bezogen sich vor allem auf die Geometrie, wobei er sich insbesondere für deren Grundlagen, die kombinatorische Topologie und die Knotentheorie interessierte. Bevor wir uns mit Reidemeisters Überlegungen befassen, soll zunächst das zugrunde liegende Phänomen erläutert werden, nämlich die Chiralität.

\section{Chiralität}

Der Chiralitätsbegriff stammt aus den Naturwissenschaften, insbesondere aus der Chemie. In der Mathematik ist er kaum geläufig und kommt auch im Buch von Reidemeister nicht vor, obschon sich sein Resultat damit in prägnanter Weise interpretieren lässt. Dass die Chiralität in der Mathematik bis anhin nur wenig Beachtung gefunden hat, erstaunt umso mehr, als es sich im Prinzip um einen rein geometrischen Begriff handelt. Mit Chiralität ist eine Reihe reizvoller Probleme verbunden, die ein grosses Anwendungspotential aufweisen. Dieser Artikel möchte dazu beitragen, hier eine interdisziplinäre Lücke zu schliessen. Bei den folgenden Ausführungen lassen wir uns ganz von der Anschauung leiten.

Das Wort Chiralität wurde erstmals 1893 vom britischen Physiker Lord Kelvin benützt, fand aber erst etwa ab 1960 geläufige Verwendung. Kelvin definierte wie folgt: „Ich nenne eine geometrische Figur oder auch Punktmenge chiral, oder sage sie habe Chiralität, wenn sie nicht mit ihrem Spiegelbild zur Deckung gebracht werden kann (... ). “ Unter der Formulierung ,zur Deckung bringen“ versteht Kelvin stillschweigend ,durch eine eigentliche Bewegung zur Deckung bringen', d.h. durch eine Rotation, Translation oder deren Verknüpfungen (Schraubungen).

Die einfachsten (räumlichen) Figuren, bei denen Chiralität möglich ist, bestehen aus vier nicht in einer Ebene liegenden Punkten; sie legen ein Tetraeder fest (Fig. 1). Der Leser überzeugt sich leicht, dass gemäss der Kelvin'schen Definition das Tetraeder $T_{1}$ chiral ist, denn beim Versuch, $T_{1}$ durch Bewegung mit seinem Spiegelbild zur Deckung zu bringen, ist bestenfalls eine Übereinstimmung von vier Kanten (mit den Längen $a$ ) zu erreichen, die beiden Kanten mit den von $a$ verschiedenen Längen $b$ und $c, b \neq c$, sind dann aber falsch zugeordnet. Das Tetraeder $T_{2}$ hingegen ist nicht chiral; man sagt es sei achiral.

In der Ebene wird Chiralität analog über die Spiegelung an einer Geraden definiert. Und mit Hilfe der Spiegelung an einer $(d-1)$-dimensionalen Hyperebene lässt sich der Begriff 

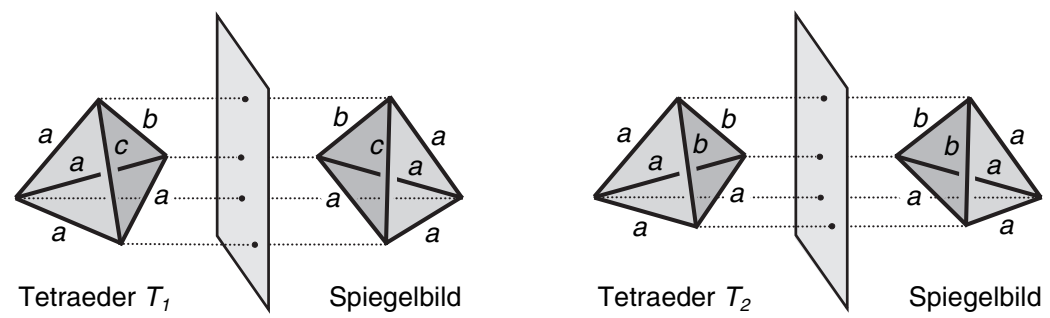

Fig. 1 Chirale und achirale Figur und deren Spiegelbilder im Raum

problemlos auf beliebige $d$-dimensionale euklidische Räume $\mathbb{R}^{d}$ mit $d \geq 1$ ausdehnen. Es muss aber betont werden, dass die Chiralität einer Figur von der Dimension des Einbettungsraums abhängt. Eine in der Ebene chirale Figur ist im Raum achiral, denn sie kann leicht durch eine räumliche Bewegung mit ihrem Spiegelbild zur Deckung gebracht werden. Oder allgemein formuliert: Eine im $\mathbb{R}^{d}$ chirale Figur verliert im $\mathbb{R}^{d+1}$ ihre Chiralität. Zwei chirale spiegelbildliche Figuren oder zwei Figuren, die je durch eigentliche Bewegungen aus solchen hervorgehen, werden als enantiomer (manchmal enantiomorph) bezeichnet.

Asymmetrische Figuren sind stets chiral, aber nicht umgekehrt. Eine Figur ist genau dann chiral, wenn sie nur eigentliche, also keine uneigentlichen Symmetrieabbildungen (Deckabbildungen) besitzt. Bei chiralen Figuren der Ebene etwa können deshalb als Symmetrieabbildungen Rotationen und Translationen vorhanden sein (Fig. 2), jedoch keine Schub- oder (als Spezialfall) Achsenspiegelungen.

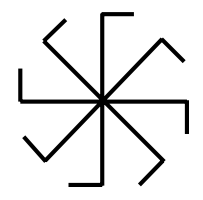

Chirale rotationssymmetrische Figur

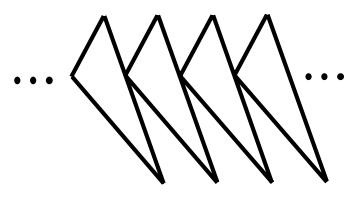

Chirale translationssymmetrische Figur (beidseitig unbegrenzt fortgesetztes Band)

Fig. 2 Chirale Figuren der Ebene mit Symmetrie

\section{Reidemeisters Kritik}

Nach dieser kurzen Darlegung des Chiralitätsbegriffs kehren wir zurück zum Kant'schen Paradoxon. Mit welchen Argumenten tritt nun der Mathematiker Reidemeister dem Philosophen Kant entgegen? Bei Reidemeister ist zu lesen (Zitat): „Die Axiome der Euklidischen Geometrie lassen sich als Aussagen über Abstände von Punkten formulieren, jeder geometrische Satz ist gleichwertig mit einer Aussage über Abstände von Punkten und Leibniz hatte recht, die geometrischen Eigenschaften der Dinge mit der Lage der Teile der Dinge gleichzusetzen. “ Insbesondere lässt sich deshalb, und hier übt Reidemeister Kritik 
an Kants Argument für den absoluten Raum, der Unterschied enantiomerer Figuren auf Abstände zurückführen. Wir wollen nicht die mathematischen Gedankengänge nachvollziehen, die Reidemeister zu diesem Schluss führen, sondern deuten sein Resultat mit Hilfe des Chiralitätsbegriffs.

Gegeben seien drei chirale Figuren, zwei davon enantiomer; sie seien mit $A, A^{\prime}$ (z.B. bei Kant die beiden Hände) und $B$ (z.B. bei Kant eine Körperhälfte) bezeichnet. Beim Vergleich der Abstände zwischen $A$ und $B$ mit den Abständen zwischen $A^{\prime}$ und $B$ zeigt sich nun ein Unterschied. Wir präzisieren dies vorerst anhand eines Beispiels in der Ebene, das zugleich den anschliessenden Satz 3.1 illustrieren soll: Betrachtet man die dreipunktigen chiralen Figuren $A=\left\{a_{1}, a_{2}, a_{3}\right\}, A^{\prime}=\left\{a_{1}^{\prime}, a_{2}^{\prime}, a_{3}^{\prime}\right\}$ und $B=\left\{b_{1}, b_{2}, b_{3}\right\}$, die je die Ecken eines ungleichseitigen Dreiecks bilden (Fig. 3), so sind für mindestens ein Indexpaar $(i, j)$ mit $i, j \in\{1,2,3\}$ die Abstände $\overline{a_{i} b_{j}}$ und $\overline{a_{i}^{\prime} b_{j}}$ verschieden (siehe auch [8]).

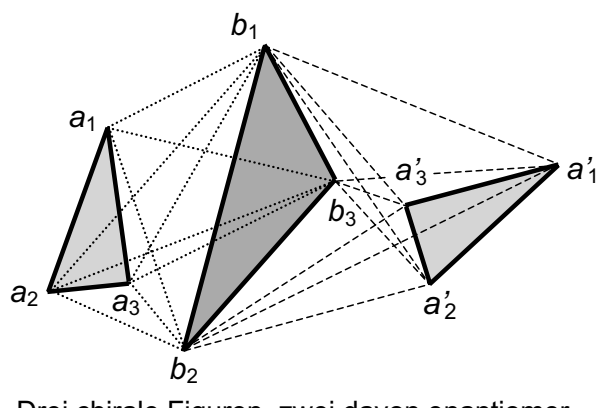

Drei chirale Figuren, zwei davon enantiomer

Fig. 3 Illustration von Satz 3.1

Bei der allgemeinen Formulierung dieses Sachverhaltes ist zu berücksichtigen, dass die chiralen Figuren $A, A^{\prime}$ und $B$ nicht asymmetrisch zu sein brauchen. Sie können, wie bereits erwähnt, nebst der Identität noch weitere Symmetrieabbildungen aufweisen, die jedoch eigentlich sein müssen.

Satz 3.1. Es seien $A$ und $A^{\prime}$ zwei enantiomere Figuren und $B$ eine weitere chirale Figur des $\mathbb{R}^{d}$. Ferner sei $\varepsilon$ eine Isometrie, die A auf $A^{\prime}$ abbildet, und $\sigma$ eine Symmetrieabbildung von $B$. Dann existieren $a \in A$ und $b \in B$ mit $\overline{a b} \neq \overline{\varepsilon(a) \sigma(b)}$.

Beweis (indirekt). Angenommen, es gilt $\overline{a b}=\overline{\varepsilon(a) \sigma(b)}$ für alle $a \in A$ und $b \in B$. In diesem Falle gibt es eine Isometrie $\phi$ mit $\phi(a)=\varepsilon(a)$ für alle $a \in A$ und $\phi(b)=\sigma(b)$ für alle $b \in B$. Weil aber $A, A^{\prime}$ und $B$ als chirale Figuren je mindestens $d+1$ Punkte in allgemeiner Lage besitzen, und eine Isometrie des $\mathbb{R}^{d}$ durch $d+1$ Punkte in allgemeiner Lage und deren Bildpunkte eindeutig festgelegt ist, folgt $\phi=\varepsilon=\sigma$. Dies steht jedoch im Widerspruch dazu, dass $\sigma$ eigentlich und $\varepsilon$ uneigentlich ist.

Die Quintessenz unserer in Satz 3.1 formulierten Interpretation des Resultats von Reidemeister lautet: Enantiomere Figuren $A$ und $A^{\prime}$ bringen dann einen Abstandsunterschied hervor, wenn der metrische Bezug zu einer weiteren chiralen Figur $B$ hergestellt wird. Am 
Ende seiner Arbeit fügt Reidemeister hinzu, dass Kants Paradoxon nicht entstanden wäre, wenn dieser seinen absoluten Raum mit einem Koordinatensystem ausgestattet hätte. Damit bringt Reidemeister zum Ausdruck, dass in der Mathematik zur Unterscheidung zweier enantiomerer Figuren $A$ und $A^{\prime}$ als chirale Bezugsfigur $B$ gewöhnlich ein Koordinatensystem verwendet wird. Soviel zur Analyse des Kant'schen Paradoxons. Welche praktische Bedeutung dem Resultat dieser Analyse zukommt, soll nachfolgend erläutert werden.

\section{Diachiralität}

Wir nehmen an, unsere enantiomeren Figuren $A$ und $A^{\prime}$ sowie die chirale Figur $B$ seien (dreidimensionale) Objekte im Alltag oder bei Naturvorgängen. Der Einfachheit halber beschränken wir uns auf starre Objekte. Gewisse interne Beweglichkeiten, etwa bei Händen oder Molekülen, sollen vernachlässigt bzw. durch ihren dynamischen Durchschnitt ersetzt werden. Externe Bewegungen, also Bewegungen der Objekte als Ganzes, sind zugelassen. Unter diesen Voraussetzungen bezeichnen wir den Sachverhalt, wonach bei jeder gegenseitigen Lage der Objekte $A, A^{\prime}$ und $B$ der Satz 3.1 zutrifft, als Diachiralität.

Der aufgrund von Diachiralität stets vorhandene Abstandsunterschied hat zur Folge, dass gewisse Wechselwirkungen zwischen $A$ und $B$ verschieden sein müssen von den entsprechenden Wechselwirkungen zwischen $A^{\prime}$ und $B$. Es muss also immer eine Beobachtung geben, bei der ein Unterschied sichtbar wird. Ein in der Chemiegeschichte wichtiges Beispiel hiefür ist die sogenannte optische Aktivität: Zwei enantiomere, phasengleich zirkular polarisierte, monochromatische Lichtstrahlen $A$ und $A^{\prime}$ gehen verschiedene Wechselwirkungen mit einem chiralen Molekül $B$ ein, was eine Drehung der Schwingungsebene des resultierenden planpolarisierten Lichtes bewirkt; man spricht von der optischen Drehung des Moleküls. Dabei entstehen bei gleicher Wellenlänge für enantiomere Moleküle entgegengesetzte Drehwinkel. Auf dieser Basis entdeckte der Chemiker L. Pasteur 1848 als erster das Phänomen der Chiralität bei Molekülen; er bezeichnete seine Beobachtung als Dissymmetrie.

Wichtig ist Diachiralität u.a. in der pharmazeutischen Chemie: Enantiomere Moleküle $A$ und $A^{\prime}$, die in einem Medikament enthalten sein können, verhalten sich zu einem chiralen Rezeptor $B$ wie zwei enantiomere Schlüssel zu einem chiralen Schloss. Passt eines der beiden Enantiomere $A$ oder $A^{\prime}$ zu $B$, geht es also eine physiologische Wechselwirkung ein, so tut es das andere Enantiomer aufgrund von Diachiralität beim gleichen Rezeptor nicht. In seltenen Fällen kann es passieren, dass die beiden Enantiomere zwei verschiedene Rezeptoren ansprechen, was dann zu unterschiedlichen physiologischen Reaktionen führt. Contergan (Thalidomid), das zwischen 1957 und 1961 als Gemisch von Enantiomeren (Fig. 4) auf dem Markt war, ist ein klassisches Beispiel. Während eines der Enantiomere (R-Thalidomid) zwar wie erwünscht den Schlaf fördert, bewirkt das andere (S-Thalidomid) schwere Missbildungen bei Neugeborenen (R und S bezeichnen den Orientierungssinn gemäss Abschnitt 6). Ausgelöst durch den Contergan-Skandal hat in den letzten Jahrzehnten die ,chirale Synthesechemie‘ zunehmend an Bedeutung gewonnen. 
<smiles>O=C1CC[C@H](N2C(=O)c3ccccc3C2=O)C(=O)N1</smiles>

R-Thalidomid (Schlaf fördernd)<smiles>C[C@@H](CCC(N)=O)C1CCC(=O)N1C</smiles>

S-Thalidomid

(Missbildungen auslösend)

Fig. 4 Enantiomere Moleküle mit unterschiedlicher physiologischer Wirkung

\section{Chiralitätsklassen}

Eine Menge chiraler Figuren in zwei Chiralitätsklassen einteilen heisst, aus allen Figuren zwei Teilmengen so zu bilden, dass nie enantiomere Figuren derselben Teilmenge angehören. Sind diese Figuren Objekte des täglichen Lebens, werden die Klassen meist mit ,rechts‘ und ,links` bezeichnet; welche Namen man wählt, ist aber letztlich belanglos. Chiralitätsklassen sind sowohl im Alltags- als auch im Wissenschaftsbereich bei der Kommunikation unerlässlich: „Ich habe meinen linken Handschuh verloren“ oder „In diesem Fläschchen befindet sich R-Thalidomid“. Solche Aussagen beziehen sich auf eine Einteilung chiraler Objekte in Chiralitätsklassen. Das Bilden von Chiralitätsklassen basiert immer auf Diachiralität. Beispielsweise betrachten wir von zwei enantiomeren Schuhen $A$ und $A^{\prime}$ eines Paars den einen Schuh als zum Fuss $B$ gehörig, weil wir unbewusst bei jeder gegenseitigen Lage Abstände zwischen $A$ und $B$ wahrnehmen, die sich von den entsprechenden Abständen zwischen $A^{\prime}$ und $B$ unterscheiden.

In chiralen Objekten der Natur findet man häufig eine gemeinsame chirale Grobstruktur, etwa eine Spirale, die dann zur Bildung von Chiralitätsklassen benützt wird. So gehören ein Schneckenhaus, eine Bohnenpflanze oder auch eine Doppelhelix in die gleiche Chiralitätsklasse, wenn die festgestellten Spiralen den gleichen Spiralsinn haben (beachte, dass eine Spirale von beiden axialen Seiten betrachtet werden kann, der Spiralsinn ändert sich nicht). Nebenbei bemerkt sind Weinbergschnecken (Fig. 5) gewöhnlich rechtsdrehend; nur eine unter Tausenden macht da eine Ausnahme, und es soll französische FeinschmeckerLokale geben, die einem die Zeche erlassen, wenn man eine davon auf dem Teller entdeckt.

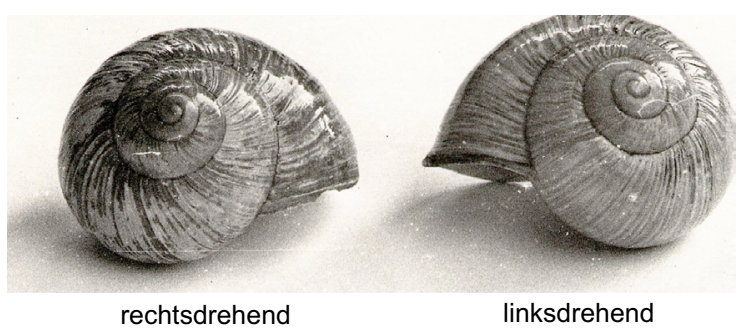

Fig. 5 Enantiomere Weinbergschnecken (linksdrehende selten) 


\section{Orientierung}

Bei vielen Mengen chiraler Objekte ist jedoch schwerlich eine gemeinsame chirale Grobstruktur erkennbar, die zur Bildung von Chiralitätsklassen benützt werden kann. Wie sind ,kartoffelförmige' chirale Figuren oder ,unförmige' chirale Moleküle zum Beispiel mit einer Spirale oder einer Hand in Verbindung zu bringen? Im Prinzip braucht es ein Enantiomerie-erhaltendes Verfahren, das komplizierte chirale Objekte auf eine gemeinsame chirale Struktur zurückführt. Damit das Verfahren für alle Objekte der betrachteten Menge in gleicher Weise Anwendung findet, muss es durch eindeutige Regeln, d.h. durch einen Algorithmus, festgelegt sein. Ein solches Verfahren nennen wir eine Orientierung der betrachteten Menge chiraler Objekte; bei den für die beiden Chiralitätsklassen vereinbarten Namen (oder Symbolen) sprechen wir von Orientierungssinn.

Ein wichtiges Beispiel einer Orientierung in der Chemie ist ein von Cahn, Ingold und Pre$\log$ in den 1950er Jahren ausgearbeitetes Verfahren, welches heute in Anlehnung an ihre Namen als ,CIP-Methode' bezeichnet wird; für den Orientierungssinn werden die Symbole R (rectus) und S (sinister) verwendet. Die CIP-Methode, die wir hier nicht besprechen können, hat sich in der Praxis bewährt, u.a. deshalb, weil sie sich auf gängige Strukturkonzepte bezieht. V. Prelog von der ETH Zürich erhielt 1975 den Chemie-Nobelpreis, auch für seine Verdienste in diesem Zusammenhang [8].

An der Universität Zürich entwickelten die Autoren und andere ab 1970 ein Verfahren, das aus einer intern beweglichen chemischen Struktur, basierend auf einem streng mathematischen Modell, einen eindeutigen Namen sowie die Symmetriegruppe generiert. Der zugrunde liegende Algorithmus stellt ausserdem fest, ob die Struktur chiral oder achiral ist, und nimmt im chiralen Fall eine Orientierung vor [4]. Die Vorgehensweise stützt sich auf keine der bisherigen Strukturkonzepte und eignet sich für eine Computerimplementation. ${ }^{1}$

Im zweiten Teil dieser Arbeit wollen wir das Prinzip dieses Verfahrens am Beispiel von konvexen Polytopen erläutern und damit gleichzeitig bisher Gesagtes verdeutlichen und konkretisieren. Konvexe Polytope sind dafür besonders geeignet, weil sie wie chemische Strukturen auf endlichen Punktmengen basieren.

\section{Konvexe Polytope}

Der Begriff Polytop ist die $d$-dimensionale Verallgemeinerung der Begriffe Polygon für $d=2$ und Polyeder für $d=3$ (Fig. 6). Wir beschränken uns hier auf konvexe Polytope, da sich in diesem Spezialfall vieles einfacher darstellen lässt; das Verfahren liesse sich jedoch auch auf nicht konvexe Polytope ausdehnen. Konvexe Polytope können auf verschiedene Arten definiert werden [5, 14]; wir wählen eine Definition, die im Hinblick auf unsere Zielsetzung möglichst zweckmässig ist:

Definition 7.1. Die konvexe Hülle $P$ einer endlichen Punktmenge des euklidischen Raumes $\mathbb{R}^{d}(d \geq 1)$ mit mindestens $d+1$ Punkten in allgemeiner Lage heisst konvexes d-dimensionales Polytop. Der Einfachheit halber sagen wir gewöhnlich kurz Polytop. Ein

\footnotetext{
${ }^{1}$ Dieses Projekt wurde ab 1975 unterstützt vom ,Schweizerischen Nationalfonds zur Förderung der Wissenschaftlichen Forschung
} 


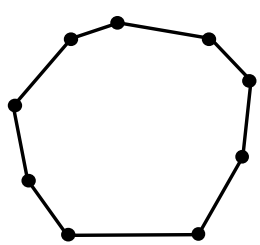

Polygon

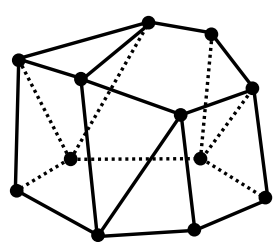

Polyeder

Fig. 6 Zweidimensionales und dreidimensionales Polytop

Punkt eines Polytops $P$, der bezüglich jedes Liniensegmentes von $P$ (Verbindungsstrecke zweier Punkte von $P$ ) höchstens Randpunkt ist, heisst Ecke von $P$; die Ecken bilden die Eckenmenge $X$ von $P$.

\section{Bemerkungen}

(1) Ein $d$-dimensionales Polytop wird hier stets im $\mathbb{R}^{d}$ und nicht in einem höher dimensionalen Einbettungsraum betrachtet, was im Zusammenhang mit Chiralität wichtig ist.

(2) Ein Polytop besteht als konvexe Hülle nicht nur aus dem Rand, sondern ebenso aus dem Innern. So ist etwa ein Polygon eine Fläche oder ein Polyeder ein Körper, auch wenn dies in den Figuren nicht zum Ausdruck kommt. Im Rahmen dieser Arbeit ist es allerdings unwichtig, ob das Innere zum Polytop gezählt wird oder nicht.

(3) Ohne Beweis sei betont, dass ein Polytop bereits durch die konvexe Hülle der Eckenmenge $X$ festgelegt ist. Bezüglich der ursprünglich vorgegebenen endlichen Punktmenge bildet $X$ eine Teilmenge mit mindestens $d+1$ Ecken in allgemeiner Lage. Beispielsweise ist das einfache Polygon, nämlich das gleichschenklige Dreieck $D r$ (Fig. 7), welches als konvexe Hülle einer 7-elementigen Punktmenge definiert ist, schon bestimmt durch die Eckenmenge $X=\{a, b, c\}$. Mit Verfahren der algorithmischen Geometrie lässt sich $X$ aus der ursprünglichen Punktmenge generieren $[1,7]$. In dieser Arbeit gehen wir stets von einer bereits vorliegenden Eckenmenge aus.

(4) Im Folgenden sprechen wir bei Bedarf von einem koordinaten-abhängigen Polytop, wenn die kartesischen Koordinaten seiner Ecken gegeben sind, und von einem koordinaten-freien Polytop bei alleiniger Vorgabe aller Abstände zwischen den Ecken. Letzteres ist nur bis auf Isometrie bestimmt.

Ein koordinaten-freies Polytop liegt insbesondere dann vor, wenn es in Form einer relationalen Darstellung oder kurz Darstellung gegeben ist. Was wir unter diesem Begriff verstehen, soll zunächst am Beispiel unseres Dreiecks $D r$ erläutert werden. Es geht darum, die durch die Eckenmenge $X=\{a, b, c\}$ festgelegte Metrik in bestimmter Weise zu beschreiben. Dabei geben wir jede Eckenverbindung durch die zwei zugehörigen zueinander symmetrischen Eckenpaare an, und fassen anschliessend die Eckenpaare von isometrischen Eckenverbindungen in sogenannten Metrikrelationen zusammen. Es resultiert so ein 3-Tupel: Zuerst steht die Eckenmenge $X$, dann folgen zwei nach den Abstandsquadraten 
4 und 9 geordnete Metrikrelationen $R_{4}$ und $R_{9}$. Dieses 3-Tupel nennen wir Darstellung von $\mathrm{Dr}$ und schreiben dafür $\operatorname{Dar}(\mathrm{Dr})$ (aus Gründen der Lesbarkeit sind bei Eckenpaaren Klammern und Kommas weggelassen):

$$
\operatorname{Dar}(D r)=\left(X, R_{4}, R_{9}\right)=\left(\{a, b, c\},\{a c, c a, b c, c b\}_{4},\{a b, b a\}_{9}\right) .
$$

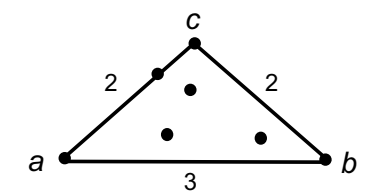

gleichschenkliges Dreieck $\mathrm{Dr}$

Fig. 7 Polygon mit der Eckenmenge $\{a, b, c\}$

In gleicher Weise wie in diesem Beispiel kann man bei einem beliebigen Polytop vorgehen. Ob man die Abstandsquadrate oder die Abstände selbst zur Festlegung der Ordnung der Metrikrelationen verwendet, spielt aufgrund der Monotonie der (auf $\mathbb{R}^{+}$definierten) Quadratfunktion keine Rolle.

Definition 7.2. Es sei $P$ ein Polytop mit der Eckenmenge $X$, und es seien $t_{1}, t_{2}, \ldots, t_{k}$ die auftretenden Abstandsquadrate zwischen je zwei verschiedenen Ecken, wobei $t_{i}<t_{j}$ für $i<j$. Das $(k+1)$-Tupel $\operatorname{Dar}(P)=\left(X, R_{t_{1}}, R_{t_{2}}, \ldots, R_{t_{k}}\right)$ mit den Metrikrelationen $R_{t_{i}}=\left\{p q \in X^{2} \mid \overline{p q}^{2}=t_{i}\right\}$ für $1 \leq i \leq k$ heisst Darstellung von $P$.

Als nächstes betrachten wir die Automorphismen der Darstellung eines Polytops, die in der Folge eine zentrale Rolle spielen; wir sprechen kurz von den Automorphismen des Polytops.

Definition 7.3. Ein Automorphismus $\alpha$ eines Polytops $P$ mit der Darstellung $\operatorname{Dar}(P)=$ $\left(X, R_{t_{1}}, R_{t_{2}}, \ldots, R_{t_{k}}\right.$ ) ist eine bijektive Abbildung der Eckenmenge $X$ auf sich selbst, welche die Eckenpaare jeder Metrikrelation $R_{t_{i}}$ mit $1 \leq i \leq k$ in die Eckenpaare derselben Metrikrelation $R_{t_{i}}$ überführt. Die Automorphismen mit dem Hintereinanderausführen als Verknüpfung bestimmen eine Gruppe, die Automorphismengruppe von $P$.

\section{Minimierungskonzept}

Bevor wir die Automorphismengruppe eines Polytops auf Chiralitäts- und Orientierungsprobleme anwenden, soll ein Verfahren besprochen werden, welches die Automorphismen generiert. Das Verfahren basiert auf einem Minimierungskonzept, das in [12] für Gebilde beschrieben ist (Gebilde, engl. relational systems, stellen eine Verallgemeinerung der hier vorliegenden Darstellungen von Polytopen dar). Beim Minimierungskonzept wird mit Nummerierungen gearbeitet:

Definition 8.1. Ist $n$ die Anzahl Ecken der Eckenmenge $X$ eines Polytops $P$, so heisst eine bijektive Abbildung $v: X \rightarrow\{1,2, \ldots, n\}$ eine Nummerierung von $P$. 
Das Minimierungskonzept umfasst drei Stufen, bei denen alle $n$ ! Nummerierungen eines $n$ eckigen Polytops $P$ berücksichtigt werden. Die drei Stufen sind allgemein formuliert, aber für das Beispiel unseres Dreiecks $D r$ in einer Nummerierungstabelle (Tab. 1) illustriert.

\begin{tabular}{|c|c|c|c|c|c|}
\hline & $v_{i}(a)$ & $v_{i}(b)$ & $v_{i}(c)$ & $\begin{array}{c}\text { Nummern-Darstellungen } \\
v_{i}(\operatorname{Dar}(D r)) \\
\end{array}$ & $\begin{array}{c}\text { Kanonisierungen (minimale fett) } \\
\left\langle v_{i}(\operatorname{Dar}(D r))\right\rangle\end{array}$ \\
\hline$v_{1}$ & 1 & 2 & 3 & $\begin{array}{l}\left(\{1,2,3\},\{13,31,23,32\}_{4}\right. \\
\left.\{12,21\}_{9}\right)\end{array}$ & $\begin{array}{l}\left((1,2,3),(13,23,31,32)_{4}\right. \\
\left.(12,21)_{9}\right)\end{array}$ \\
\hline$v_{2}$ & 1 & 3 & 2 & $\begin{array}{l}\left(\{1,3,2\},\{12,21,32,23\}_{4},\right. \\
\left.\{13,31\}_{9}\right)\end{array}$ & $\begin{array}{l}\left((1,2,3),(12,21,23,32)_{4},\right. \\
\left.(13,31)_{9}\right)\end{array}$ \\
\hline$v_{3}$ & 2 & 1 & 3 & $\begin{array}{l}\left(\{2,1,3\},\{23,32,13,31\}_{4},\right. \\
\left.\{21,12\}_{9}\right)\end{array}$ & $\begin{array}{l}\left((1,2,3),(13,23,31,32)_{4}\right. \\
\left.(12,21)_{9}\right)\end{array}$ \\
\hline$v_{4}$ & 2 & 3 & 1 & $\begin{array}{l}\left(\{2,3,1\},\{21,12,31,13\}_{4},\right. \\
\left.\{23,32\}_{9}\right)\end{array}$ & $\begin{array}{l}\left((1,2,3),(12,13,21,31)_{4},\right. \\
\left.(23,32)_{9}\right)=\operatorname{Min}(D r)\end{array}$ \\
\hline$v_{5}$ & 3 & 1 & 2 & $\begin{array}{l}\left(\{3,1,2\},\{32,23,12,21\}_{4},\right. \\
\left.\{31,13\}_{9}\right)\end{array}$ & $\begin{array}{l}\left((1,2,3),(12,21,23,32)_{4}\right. \\
\left.(13,31)_{9}\right)\end{array}$ \\
\hline$v_{6}$ & 3 & 2 & 1 & $\begin{array}{l}\left(\{3,2,1\},\{31,13,21,12\}_{4},\right. \\
\left.\{32,23\}_{9}\right)\end{array}$ & $\begin{array}{l}\left((1,2,3),(12,13,21,31)_{4},\right. \\
\left.(23,32)_{9}\right)=\operatorname{Min}(D r)\end{array}$ \\
\hline
\end{tabular}

Tab. 1: Nummerierungstabelle für $\mathrm{Dr}$

Erste Stufe: Für jede Nummerierung $v$ von $P$ werden in der Darstellung $\operatorname{Dar}(P)$ die Buchstaben durch die zugehörigen Nummern ersetzt. Es entstehen die Nummern-Darstellungen $v(\operatorname{Dar}(P))$.

Zweite Stufe: In jeder Nummern-Darstellung $v(\operatorname{Dar}(P))$ werden die Elemente innerhalb der Eckenmenge und innerhalb jeder Metrikrelation lexikographisch, d.h. der Grösse nach, zu Tupeln geordnet. Es ergeben sich die Kanonisierungen $\langle v(\operatorname{Dar}(P))\rangle$.

Dritte Stufe: Aus allen Kanonisierungen wird eine lexikographisch kleinste gewählt. Es resultiert die minimale Kanonisierung $\operatorname{Min}(P)$.

Die minimale Kanonisierung $\operatorname{Min}(P)$ kann als eindeutiger Name des durch $\operatorname{Dar}(P)$ bestimmten, koordinaten-freien Polytops aufgefasst werden. Namen dieser Art, welche die gesamte Information der Struktur in sich tragen, sind in der Chemie nützlich. Wir nennen Nummerierungen, die zum Namen $\operatorname{Min}(P)$ führen, Minimums-Nummerierungen, oder formal ausgedrückt:

Definition 8.2. Eine Nummerierung $v$ eines Polytops $P$ heisst Minimums-Nummerierung von $P$, wenn $\langle v(\operatorname{Dar}(P))\rangle=\operatorname{Min}(P)$.

In der Nummerierungstabelle für das Dreieck $\mathrm{Dr}$ kommt die minimale Kanonisierung $\operatorname{Min}(D r)$ zweimal vor, und es gibt deshalb zwei Minimums-Nummerierungen, nämlich $v_{4}$ und $v_{6}$. Aber nicht nur die minimale, sondern auch die andern Kanonisierungen treten in der Nummerierungstabelle zweimal auf, was mit den Automorphismen von $\mathrm{Dr}$ zu tun hat. Wie man sich leicht klar macht, gilt ganz allgemein für zwei Nummerierungen $\nu$ und $\mu$ eines Polytops $P$ folgende Äquivalenz:

$$
\begin{gathered}
\langle v(\operatorname{Dar}(P))\rangle=\langle\mu(\operatorname{Dar}(P))\rangle \\
\Longleftrightarrow \quad \alpha=\mu^{-1} v \text { ist Automorphismus von } P .
\end{gathered}
$$


Die Menge aller $n$ ! Nummerierungen eines $n$-eckigen Polytops $P$ zerfällt damit in Klassen mit je gleich vielen Nummerierungen wie Automorphismen. Jede dieser Nummerierungsklassen kann unter Verwendung von (8.1) zur Bestimmung der Automorphismen benützt werden; wir arbeiten hier mit der Klasse der Minimums-Nummerierungen. Beim Dreieck $D r$ ergeben sich so aus den Minimums-Nummerierungen $v_{4}$ und $v_{6}$ die Automorphismen $\alpha_{1}=v_{4}^{-1} v_{4}$ und $\alpha_{2}=v_{4}^{-1} v_{6}$, oder in Zyklenschreibweise notiert:

$$
\alpha_{1}=(a)(b)(c), \alpha_{2}=(c)(a b) .
$$

\section{Minimierungsalgorithmus}

Beim eben besprochenen Minimierungskonzept werden alle $n$ ! Nummerierungen eines $n$ eckigen Polytops in Betracht gezogen. Ein direkt darauf basierender Algorithmus wäre also exponentiell und für grössere $n$ unbrauchbar. Wir präsentieren nun einen Algorithmus, der die minimale Kanonisierung und damit verbunden die Minimums-Nummerierungen generiert, ohne dabei alle $n$ ! Nummerierungen abzuarbeiten. Die Erläuterung dieses Minimierungsalgorithmus geschieht anhand eines fünfeckigen Polyeders, der trigonalen Bipyramide $B p$, deren Ecken auf der Oberfläche eines Würfels der Kantenlänge 2 liegen (Fig. 8). Die Darstellung von $B p$ sieht wie folgt aus:

$$
\begin{aligned}
\operatorname{Dar}(B p)= & \left\{\{a, b, c, d, e\},\{c d, d c\}_{1},\{a e, e a, d e, e d\}_{4},\{b c, c b, c e, e c\}_{5},\right. \\
& \left.\{a b, b a, a d, d a, b d, d b\}_{8},\{a c, c a\}_{9},\{b e, e b\}_{12}\right) .
\end{aligned}
$$

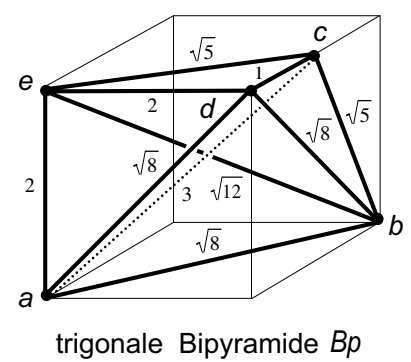

Fig. 8 Fünfeckiges Polyeder im Würfel mit Kantenlänge 2

Der Minimierungsalgorithmus ist rekursiv und funktioniert gemäss einer Algorithmustabelle (Tab. 2) salopp formuliert wie folgt: Beim Schritt 1 werden die zwei Eckenpaare der ersten Metrikrelation von $\operatorname{Dar}(B p)$ minimiert. Nach $r-1$ Schritten $(1<r \leq 5)$ liegen ,Darstellungs-Sequenzen “ mit lexikographisch kleinsten zugehörigen ,MinimumsSequenzen ' vor. Beim Schritt $r$ werden diese Darstellungs-Sequenzen durch Hinzufügen eines Eckenpaars erweitert, und zwar geschieht dies pro Darstellungs-Sequenz mit jedem noch verbleibenden Eckenpaar derselben Metrikrelation oder, falls diese schon abgearbeitet ist, mit jedem Eckenpaar der nächst folgenden Metrikrelation von $\operatorname{Dar}(B p)$. Von den so resultierenden Darstellungs-Sequenzen werden erneut jene weiter verwendet, die zu lexikographisch kleinsten Minimums-Sequenzen führen. Bereits nach dem Schritt 5 kann 
hier der Algorithmus abgebrochen werden, da nur noch eine lexikographisch kleinste Minimums-Sequenz übrig bleibt und allein die Nummer 5 fehlt. Es resultieren eine einzige Minimums-Nummerierung $v-$ der zugehörige Automorphismus $\alpha=v^{-1} v$ ist das Neutralelement - sowie die minimale Kanonisierung:

$$
v: a \rightarrow 4, b \rightarrow 5, c \rightarrow 2, d \rightarrow 1, e \rightarrow 3,
$$

$$
\begin{aligned}
\operatorname{Min}(B p)= & \left((1,2,3,4,5),(12,21)_{1},(13,31,34,43)_{4},(23,25,32,52)_{5}\right. \\
& \left.(14,15,41,45,51,54)_{8},(24,42)_{9},(35,53)_{12}\right)
\end{aligned}
$$

\begin{tabular}{|c|c|c|}
\hline Schritt $r$ & Darstellungs-Sequenzen & $\begin{array}{c}\text { Minimums-Sequenzen } \\
\text { (lexikographisch kleinste fett) }\end{array}$ \\
\hline \multirow[t]{2}{*}{1} & $((c d, \ldots)$ & $((12, \ldots)$ \\
\hline & $((d c, \ldots)$ & $((12, \ldots)$ \\
\hline \multirow[t]{2}{*}{2} & $\left((c d, d c)_{1},(\ldots\right.$ & $\left((12,21)_{1},(\ldots\right.$ \\
\hline & $\left((d c, c d)_{1},(\ldots\right.$ & $\left((12,21)_{1},(\ldots\right.$ \\
\hline \multirow[t]{8}{*}{3} & $\left((c d, d c)_{1},(a e\right.$ & $\left((12,21)_{1},(34\right.$ \\
\hline & $\left((c d, d c)_{1},(e a\right.$ & $\left((12,21)_{1},(34\right.$ \\
\hline & $\left((c d, d c)_{1},(d e\right.$ & $\left((12,21)_{1},(23\right.$ \\
\hline & $\left((c d, d c)_{1},(e d\right.$ & $\left((12,21)_{1},(32\right.$ \\
\hline & $\left((d c, c d)_{1},(a e\right.$ & $\left((12,21)_{1},(34\right.$ \\
\hline & $\left((d c, c d)_{1},(e a\right.$ & $\left((12,21)_{1},(34\right.$ \\
\hline & $\left((d c, c d)_{1},(d e, \ldots\right.$ & $\left((12,21)_{1},(13, \ldots\right.$ \\
\hline & $\left((d c, c d)_{1},(e d\right.$ & $\left((12,21)_{1},(31\right.$ \\
\hline \multirow[t]{3}{*}{4} & $\left((d c, c d)_{1},(d e, a e\right.$ & $\left((12,21)_{1},(13,43\right.$ \\
\hline & $\left((d c, c d)_{1},(d e, e a\right.$ & $\left((12,21)_{1},(13,34\right.$ \\
\hline & $\left((d c, c d)_{1},(d e, e d, \ldots\right.$ & $\left((12,21)_{1},(13,31, \ldots\right.$ \\
\hline \multirow[t]{3}{*}{5} & $\left((d c, c d)_{1},(d e, e d, a e\right.$ & $\left((12,21)_{1},(13,31,43\right.$ \\
\hline & $\left((d c, c d)_{1},(d e, e d, e a, \ldots\right.$ & $\left((12,21)_{1},(13,31,34, \ldots\right.$ \\
\hline & & $\begin{array}{l}v: d \rightarrow 1, c \rightarrow 2, e \rightarrow 3, a \rightarrow 4 \\
\text { und somit } b \rightarrow 5\end{array}$ \\
\hline
\end{tabular}

Tab. 2: Algorithmustabelle für $B p$

Auch wenn der Minimierungsalgorithmus hier nicht allgemein formuliert wird, so sollte das Beispiel seine prinzipielle Funktionsweise genügend verdeutlichen. Der Algorithmus steht in dieser Arbeit ohnehin nicht im Vordergrund und eine ausführlichere Darlegung für beliebige Gebilde findet sich in $[13]^{2}$. Zu klären wäre die Komplexität des Minimierungsalgorithmus bei der vorliegenden Anwendung auf die Darstellung von Polytopen. Im Beispiel unserer Bipyramide $B p$ ist es möglich, ihn vorzeitig abzubrechen. Allgemein sind im Maximum so viele Schritte erforderlich, wie die Gesamtzahl der Eckenpaare beträgt, also $n(n-1)$ bei $n$ Ecken, wobei die Anzahl der Darstellungs-Sequenzen pro Schritt schwankt.

\footnotetext{
${ }^{2}$ In [13] wird ,Kanonisierung ' im Sinne von ,minimaler Kanonisierung ‘ verwendet.
} 


\section{Symmetrieaspekte}

Eine Symmetrieabbildung eines $d$-dimensionalen Polytops $P$ ist bekanntlich eine Isometrie des ganzen euklidischen Raumes $\mathbb{R}^{d}$, die $P$ auf sich selbst abbildet. Im Unterschied dazu operiert ein Automorphismus von $P$ nur auf der Eckenmenge $X$. Welches ist nun der Zusammenhang zwischen den Symmetrieabbildungen und den Automorphismen?

Satz 10.1. Zu jeder Symmetrieabbildung $\sigma$ eines Polytops $P$ gibt es genau einen Automorphismus $\alpha$ von $P$ mit $\alpha=\sigma_{X}$ und umgekehrt, wobei $\sigma_{X}$ die Einschränkung von $\sigma$ auf die Eckenmenge $X$ bezeichnet.

Beweis. Eine gegebene Symmetrieabbildung $\sigma$ von $P$ bildet die Eckenmenge $X$ auf sich selbst ab. Andernfalls müsste nämlich eine Ecke, die höchstens Randpunkt eines Liniensegmentes von $P$ sein kann, in das Innere eines Liniensegmentes von $P$ übergehen, was zu Widersprüchen führt. Da nun $\sigma$ jede Eckenverbindung in eine isometrische Eckenverbindung abbildet, und damit jedes Eckenpaar in eines derselben Metrikrelation übergeht, liegt mit $\alpha=\sigma_{X}$ genau ein Automorphismus von $P$ vor. Umgekehrt gibt es bei gegebenem Automorphismus $\alpha$ von $P$ mindestens eine Isometrie $\sigma$ mit $\sigma_{X}=\alpha$. Weil aber ein Polytop $d+1$ Ecken in allgemeiner Lage besitzt, ist $\sigma$ eindeutig bestimmt (wie bereits erwähnt, wird eine Isometrie des $\mathbb{R}^{d}$ durch $d+1$ Punkte in allgemeiner Lage und deren Bildpunkte eindeutig festgelegt). Die Isometrie $\sigma$ ist zudem eine Symmetrieabbildung des ganzen Polytops $P$, da dieses konvexe Hülle der Eckenmenge $X$ ist. Es sei hinzugefügt, dass mit der eineindeutigen Zuordnung zwischen den Automorphismen und den Symmetrieabbildungen ein Gruppenisomorphismus vorliegt.

Im Beispiel unseres gleichschenkligen Dreiecks $D r$ ist die eineindeutige Zuordnung zwischen den Automorphismen (8.2) und den Symmetrieabbildungen evident: $\mathrm{Zu} \alpha_{1}$ gehört die Identität und zu $\alpha_{2}$ (da $c$ Fixelement ist) eine Achsenspiegelung. Bei der Bipyramide $B p$ entspricht dem Neutralelement als einzigem Automorphismus die Identität und $B p$ ist deshalb asymmetrisch. Im Allgemeinen gibt jedoch ein vorliegender Automorphismus keinen Aufschluss über die Art der zugehörigen Symmetrieabbildung. Das folgende Beispiel zweier Polygone mag dies illustrieren. Wir betrachten einerseits das gleichschenklige Trapez $\mathrm{Tr}$ und andererseits das Parallelogramm $\mathrm{Pa}$ (Fig. 9). In beiden Fällen lassen sich die Minimums-Nummerierungen und die Automorphismen von Hand ermitteln:

$$
\begin{gathered}
\operatorname{Tr}: \quad v_{1}: a \rightarrow 4, b \rightarrow 3, c \rightarrow 1, d \rightarrow 2, \quad v_{2}: a \rightarrow 3, b \rightarrow 4, c \rightarrow 2, d \rightarrow 1 \\
\alpha_{1}=(a)(b)(c)(d), \alpha_{2}=(a b)(c d) \\
\operatorname{Pa}: \quad v_{1}: a \rightarrow 4, b \rightarrow 1, c \rightarrow 2, d \rightarrow 3, \quad v_{2}: a \rightarrow 2, b \rightarrow 3, c \rightarrow 4, d \rightarrow 1, \\
\alpha_{1}=(a)(b)(c)(d), \alpha_{2}=(a c)(b d) .
\end{gathered}
$$

Obschon die durch (10.1) und (10.3) bestimmten Automorphismengruppen von $\operatorname{Tr}$ und $\mathrm{Pa}$ isomorphe Permutationsgruppen sind, zeigt sich bei den Symmetrieabbildungen ein Unterschied: Bei $T r$ gehört zu $\alpha_{2}$ eine Achsen-, bei $P a$ jedoch eine Punktspiegelung. Insbesondere geht demnach aus der Struktur der beiden Automorphismengruppen nicht 


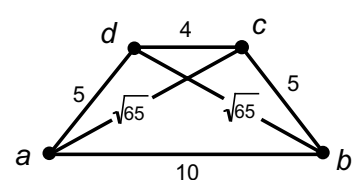

gleichschenkliges Trapez Tr

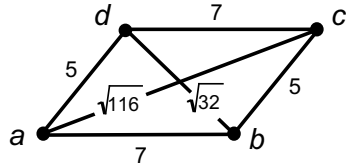

Parallelogramm $\mathrm{Pa}$

Fig. 9 Achirales und chirales Polygon mit isomorpher Automorphismengruppe

hervor, dass $\mathrm{Tr}$ achiral und $\mathrm{Pa}$ chiral ist. Im Unterschied dazu kann bei der Bipyramide $B p$ aus dem Vorhandensein nur eines Automorphismus, dem Neutralelement, auf die Chiralität geschlossen werden.

Als Chiralitäts-Test bezeichnen wir ein Verfahren, das prüft, ob ein Polytop chiral ist. Ein Chiralitäts-Test besteht zunächst aus einem Automorphismen-Test und, falls dieser nicht ausreicht, zusätzlich aus einem Simplex-Test, wie er im übernächsten Abschnitt 12 besprochen wird. Beim Automorphismen-Test versucht man allein aufgrund der Struktur der Automorphismengruppe zu entscheiden, ob Chiralität vorliegt. Wie wir soeben anhand der einfachen Polygone $T r$ und $P a$ gesehen haben, gibt es Fälle, wo dies nicht möglich ist. Interessant ist die Frage, welche notwendigen und hinreichenden Bedingungen erfüllt sein müssen, damit ein Automorphismen-Test zum Ziel führt. Wir kennen die vollständige Antwort nicht, nennen aber einen Satz, der eine einfache hinreichende Bedingung beinhaltet:

Satz 10.2. Ein Polytop ist chiral, wenn die Ordnung seiner Automorphismengruppe ungerade ist.

Beweis. Wir zeigen, dass die Ordnung der Automorphismengruppe eines achiralen Polytops gerade ist: Die Automorphismengruppe und die Symmetriegruppe eines Polytops haben dieselbe Ordnung (Satz 10.1). Letztere ist aber bei einem achiralen Polytop gerade, weil die eigentlichen Symmetrieabbildungen eine Untergruppe vom Index 2 bilden und es deshalb gleich viele eigentliche wie uneigentliche Symmetrieabbildungen gibt.

\section{Orientierte Simplexe}

In diesem Abschnitt befassen wir uns mit orientierten Simplexen, um damit den erwähnten Simplex-Test vorzubereiten.

Definition 11.1. Ein $d$-dimensionales Polytop mit $d+1$ Ecken heisst ein $d$-dimensionales Simplex. Ist zusätzlich eine Reihenfolge der Ecken festgelegt, so spricht man von einem orientierten d-dimensionalen Simplex. Wir nennen es kurz $o$-Simplex, bezeichnen es mit $S$ und schreiben die Ecken zur Festlegung der Reihenfolge in ein $(d+1)$-Tupel, etwa $S=\left(x_{0}, x_{1}, \ldots, x_{d}\right)$.

Weshalb wird von orientierten bzw. o-Simplexen gesprochen? Aufgrund der Eckenreihenfolge lassen sich alle koordinaten-abhängigen o-Simplexe gleicher Dimension in bekannter Weise mit Hilfe von Determinantenvorzeichen orientieren, und zwar auch dann, 
wenn sie im metrischen Sinne achiral sind. Dazu betrachtet man zu einem o-Simplex $S=\left(x_{0}, x_{1}, \ldots, x_{d}\right)$ die $d \times d$-Matrix $M_{S}$, deren $i$-te Zeile der Komponentenvektor $\overrightarrow{x_{0} x_{i}}$ $(1 \leq i \leq d)$ ist. Bezeichnet $\mathrm{D}$ die Determinantenfunktion, so sei $\operatorname{det}(S):=\mathrm{D}\left(M_{S}\right)$. Es gilt $\operatorname{det}(S) \neq 0$, da ein o-Simplex per definitionem nicht entartet ist. Man definiert nun: Zwei $d$-dimensionale o-Simplexe $S$ und $T$ sind gleich orientiert, falls $\operatorname{det}(S)$ und $\operatorname{det}(T)$ das gleiche Vorzeichen haben, sonst verschieden orientiert.

Ob zwei o-Simplexe $S=\left(x_{0}, x_{1}, \ldots, x_{d}\right)$ und $T=\left(y_{0}, y_{1}, \ldots, y_{d}\right)$ gleich oder verschieden orientiert sind, ist auch im Determinantenvorzeichen einer einzigen $d \times d$-Matrix $M_{S, T}$ verborgen, welche die Abstände zwischen $S$ und $T$ berücksichtigt:

$$
\begin{gathered}
M_{S, T}=\left(\begin{array}{cccc}
m_{11} & m_{12} & \cdots & m_{1 d} \\
m_{21} & m_{22} & \cdots & m_{2 d} \\
\vdots & \vdots & \ddots & \vdots \\
m_{d 1} & m_{d 2} & \cdots & m_{d d}
\end{array}\right) \\
\operatorname{mit} m_{i j}=1 / 2 \cdot\left({\overline{x_{0} y_{j}}}^{2}+{\overline{x_{i} y_{0}}}^{2}-{\overline{x_{i} y_{j}}}^{2}-{\overline{x_{0} y_{0}}}^{2}\right) \text { für } i, j \in\{1,2, \ldots, d\} .
\end{gathered}
$$

Setzt man $\operatorname{det}(S, T):=\mathrm{D}\left(M_{S, T}\right)$, so gilt:

Satz 11.1. Zwei d-dimensionale o-Simplexe $S$ und $T$ sind genau dann gleich orientiert, wenn $\operatorname{det}(S, T)$ positiv ist. ${ }^{3}$

Beweis. Wir zeigen, dass die Beziehung

$$
\operatorname{det}(S, T)=\operatorname{det}(S) \operatorname{det}(T)
$$

erfüllt ist, der Satz ist dann bewiesen. Dazu bilden wir zunächst das Matrixprodukt $M_{S} M_{T}^{*}$, wobei $M_{T}^{*}$ die transponierte Matrix von $M_{T}$ bezeichnet. Für das $(i j)$-te Element $p_{i j}$ dieses Produkts resultiert ein Skalarprodukt, das sich u.a. mit Hilfe der Vektoridentität $\overrightarrow{a b} \cdot \overrightarrow{a c}=$ $1 / 2 \cdot\left(\overline{a b}^{2}+\overline{a c}^{2}-\overline{b c}^{2}\right)$ (Kosinussatz) umformen lässt:

$$
\begin{aligned}
p_{i j} & =\overrightarrow{x_{0} x_{i}} \cdot \overrightarrow{y_{0} y_{j}}=\overrightarrow{x_{0} x_{i}} \cdot\left(\overrightarrow{x_{0} y_{j}}-\overrightarrow{x_{0} y_{0}}\right)=\overrightarrow{x_{0} x_{i}} \cdot \overrightarrow{x_{0} y_{j}}-\overrightarrow{x_{0} x_{i}} \cdot \overrightarrow{x_{0} y_{0}} \\
& =1 / 2 \cdot\left({\overline{x_{0} x_{i}}}^{2}+{\overline{x_{0} y_{j}}}^{2}-{\overrightarrow{x_{i} y_{j}}}^{2}\right)-1 / 2 \cdot\left({\overline{x_{0} x_{i}}}^{2}+{\overrightarrow{x_{0} y_{0}}}^{2}-{\overrightarrow{x_{i} y_{0}}}^{2}\right) \\
& =1 / 2 \cdot\left({\overline{x_{0} y_{j}}}^{2}+{\overline{x_{i} y_{0}}}^{2}-{\overrightarrow{x_{i} y_{j}}}^{2}-{\overrightarrow{x_{0} y_{0}}}^{2}\right) .
\end{aligned}
$$

Der letzte Term zeigt, dass $p_{i j}=m_{i j}$ gemäss (11.1) und somit $M_{S, T}=M_{S} M_{T}^{*}$. Damit ergibt sich schliesslich:

$$
\operatorname{det}(S, T)=\mathrm{D}\left(M_{S, T}\right)=\mathrm{D}\left(M_{S} M_{T}^{*}\right)=\mathrm{D}\left(M_{S}\right) \mathrm{D}\left(M_{T}\right)=\operatorname{det}(S) \operatorname{det}(T) .
$$

\footnotetext{
${ }^{3}$ Die Idee für diesen Satz stammt aus einer unveröffentlichten Arbeit von Dimitrios Pazis (National Technical University of Athens), die er im Rahmen des unter Fussnote 1 erwähnten Nationalfondsprojekts geschrieben hat.
} 
Es resultiert auch eine Beziehung über den Betrag von $\operatorname{det}(S, T)$ :

Korollar 11.1. Bezeichnen $A_{S}$ und $A_{T}$ die Inhalte zweier d-dimensionaler o-Simplexe $S$ und $T$, so gilt:

$$
|\operatorname{det}(S, T)|=(d !)^{2} A_{S} A_{T} .
$$

Beweis. (11.3) folgt unmittelbar, wenn man in (11.2) beidseitig den Betrag bildet und dabei benützt, dass bekanntlich $|\operatorname{det}(S)|=d ! A_{S}$ bzw. $|\operatorname{det}(T)|=d ! A_{T}$ ist.

Wir illustrieren die Resultate mit zwei koordinaten-freien o-Dreiecken (Fig. 10), nämlich $S=\left(x_{0}, x_{1}, x_{2}\right)$ und $T=\left(y_{0}, y_{1}, y_{2}\right)$. Zur Bestimmung der Matrix $M_{S, T}$ gemäss (11.1) werden die folgenden Abstandsquadrate benötigt:

$$
\begin{array}{lll}
{\overline{x_{0} y_{0}}}^{2}=194, & {\overline{x_{0} y_{1}}}^{2}=169, & {\overline{x_{0} y_{2}}}^{2}=625, \\
{\overline{x_{1} y_{0}}}^{2}=74, & {\overline{x_{1} y_{1}}}^{2}=49, & {\overline{x_{1} y_{2}}}^{2}=361, \\
{\overline{x_{2} y_{0}}}^{2}=101, & {\overline{x_{2} y_{1}}}^{2}=116, & {\overline{x_{2} y_{2}}}^{2}=500 .
\end{array}
$$

Damit ergibt sich $M_{S, T}=\left(\begin{array}{cc}0 & 72 \\ -20 & 16\end{array}\right)$ und daraus $\operatorname{det}(S, T)=1440$.

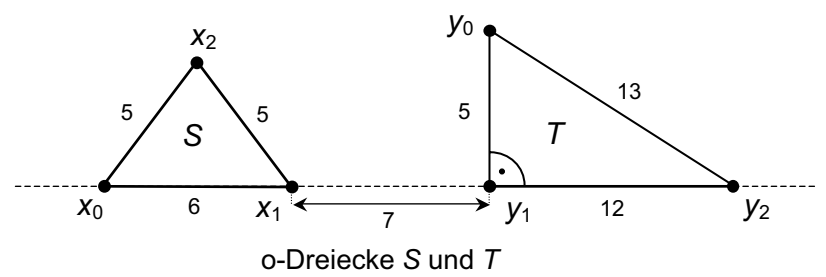

Fig. 10 Gleichorientierte zweidimensionale o-Simplexe

Da die Determinante $\operatorname{det}(S, T)$ positiv ist, sind wegen Satz 11.1 die beiden o-Dreiecke $S$ und $T$ gleich orientiert. Ihr Betrag lässt sich auch unter Verwendung von Korollar 11.1 berechnen: Aus $d=2, A_{S}=12$ und $A_{T}=30$ erhält man $|\operatorname{det}(S, T)|=1440$. Die Determinante $\operatorname{det}(S, T)$ selbst hat den Wert 1440 bei gleicher und -1440 bei verschiedener Orientierung der o-Dreiecke $S$ und $T$, unabhängig von ihrer gegenseitigen Lage. Es sei beigefügt, dass die beiden Vorzeichen von $\operatorname{det}(S, T)$ eine Folge von Satz 3.1 sind, und zwar in einem erweiterten Sinne: Das metrisch achirale Dreieck $S$ erhält nämlich seine Chiralität erst durch die unterschiedlichen Individualisierungen der Ecken. Auf diesen im Zusammenhang mit chemischen Modellvorstellungen wichtigen Sachverhalt wird hier nicht weiter eingegangen.

\section{Chiralität von Polytopen}

Wie sieht nun der Simplex-Test aus, wie lässt sich also feststellen, ob ein Polytop chiral ist, wenn der Automorphismen-Test nicht ausreicht? Wir arbeiten mit einem o-Simplex $S=\left(x_{0}, x_{1}, \ldots, x_{d}\right)$ eines $d$-dimensionalen Polytops $P$, d.h. die Ecken von $S$ sind jetzt 
speziell Ecken von $P$. Nebst $S$ betrachten wir für einen Automorphismus $\alpha$ von $P$ auch das isometrische o-Simplex $\alpha(S)=\left(\alpha\left(x_{0}\right), \alpha\left(x_{1}\right), \ldots, \alpha\left(x_{d}\right)\right)$ von $P$. Die beiden o-Simplexe $S$ und $\alpha(S)$ sind genau dann gleich orientiert, wenn die zu $\alpha$ gehörige Symmetrieabbildung eigentlich ist, und zwar unabhängig vom betrachteten o-Simplex $S$ von $P$. Beim Simplex-Test dürfen wir wegen Satz 10.2 von einem $P$ ausgehen, dessen Ordnung $p$ der Automorphismengruppe gerade ist. Und weil bei chiralem $P$ alle $p$ Symmetrieabbildungen und bei achiralem $P$ deren $p / 2$ eigentlich sind, ergibt sich schliesslich mit Benützung von Satz 11.1:

Simplex-Test. Es sei $P$ ein Polytop mit gerader Ordnung p der Automorphismengruppe. Für den Chiralitätsnachweis wählt man von $P$ ein o-Simplex $S$ sowie $p / 2$ vom Neutralelement verschiedene Automorphismen $\alpha$ und zeigt, dass die Determinante $\operatorname{det}(S, \alpha(S))$ stets positiv ist.

\section{Bemerkungen}

(1) Bei koordinaten-abhängigem $P$ ist es zweckmässig, $\operatorname{det}(S, \alpha(S))$ nicht über die Matrix $M_{S, \alpha(S)}$ gemäss (11.1), sondern mit $\operatorname{det}(S)$ und $\operatorname{det}(\alpha(S))$ zu berechnen. Aufgrund von (11.2) gilt ja $\operatorname{det}(S, \alpha(S))=\operatorname{det}(S) \operatorname{det}(\alpha(S))$.

(2) Häufig ist es nicht notwendig, $p / 2$ vom Neutralelement verschiedene Automorphismen zu betrachten. Liegt beispielsweise eine zyklische Automorphismengruppe vor, so kann man sich auf ein erzeugendes Element beschränken. Auf die Frage, wie Beschränkungen dieser Art im allgemeinen Fall aussehen, wird hier nicht eingegangen.

Der Simplex-Test soll jetzt bei den koordinaten-freien Polygonen $\mathrm{Tr}$ und $\mathrm{Pa}$ durchgeführt werden. In beiden Fällen wählen wir das o-Dreieck $S=(a, b, c)$ (siehe Fig. 9). Mit dem einzigen zu betrachtenden Automorphismus $\alpha_{2}$ von (10.1) bzw. (10.3) ergibt sich durch Rechnung:

$$
\begin{aligned}
& T r: \operatorname{det}\left(S, \alpha_{2}(S)\right)=-1600, \text { also } \operatorname{Tr} \text { achiral, } \\
& P a: \operatorname{det}\left(S, \alpha_{2}(S)\right)=784, \text { also } P a \text { chiral. }
\end{aligned}
$$

Da 1-dimensionale Polytope - es handelt sich um Strecken - immer achiral sind, ist der Chiralitätsbegriff erst bei Polygonen, Polyedern und Polytopen höherer Dimension von Interesse. Wir wollen an dieser Stelle kurz ein 4-dimensionales Polytop hinsichtlich Chiralität untersuchen. Das koordinaten-abhängige Polytop, es sei mit Pt bezeichnet, ist definiert als konvexe Hülle der folgenden 7 Punkte:

$$
\begin{aligned}
& a=(3,2,5,3), \quad b=(3,2,1,1), \quad c=(3,6,1,3), \quad d=(4,4,5,4), \\
& e=(7,3,3,4), \quad f=(5,6,2,4), \quad g=(7,2,1,3) \text {. }
\end{aligned}
$$

Man verifiziert: $S=(a, b, c, d, e)$ ist wegen $\operatorname{det}(S) \neq 0$ nicht entartet, d.h. die 5 Punkte $a$, $b, c, d$ und $e$ befinden sich in allgemeiner Lage; mit $P t$ liegt demnach ein 4-dimensionales Polytop vor. Zudem gehören alle 7 Punkte zur Oberfläche einer 4-dimensionalen $\mathrm{Ku}-$ gel mit Mittelpunkt $(3,2,1,6)$ und Radius 5; sie bilden deshalb die Ecken von Pt. Aus der Darstellung von $P t$ ergeben sich mit dem Minimierungsalgorithmus ${ }^{4}$ die Minimums-

\footnotetext{
${ }^{4}$ Das hier verwendete Programm stammt von Ralf Gugisch (Universität Bayreuth).
} 
Nummerierungen. Und weil drei Minimums-Nummerierungen und somit auch drei Automorphismen vorhanden sind, ist $P t$ schon aufgrund des Automorphismen-Tests chiral (Satz 10.2); es braucht den Simplex-Test nicht. Die Minimums-Nummerierungen sehen wie folgt aus:

$$
\begin{aligned}
& v_{1}: a \rightarrow 2, b \rightarrow 7, c \rightarrow 4, d \rightarrow 1, e \rightarrow 5, f \rightarrow 3, g \rightarrow 6 \\
& v_{2}: a \rightarrow 4, b \rightarrow 7, c \rightarrow 6, d \rightarrow 3, e \rightarrow 1, f \rightarrow 5, g \rightarrow 2, \\
& v_{3}: a \rightarrow 6, b \rightarrow 7, c \rightarrow 2, d \rightarrow 5, e \rightarrow 3, f \rightarrow 1, g \rightarrow 4
\end{aligned}
$$

\section{Orientierung chiraler Polytope}

Auf der Grundlage des Minimierungskonzepts soll nun eine Orientierung der chiralen Polytope gleicher Dimension definiert werden. Dazu gehen wir von einer MinimumsNummerierung $v$ eines chiralen $d$-dimensionalen Polytops $P$ aus. Für jedes o-Simplex $S=\left(x_{0}, x_{1}, \ldots, x_{d}\right)$ von $P$ denke man sich das zugehörige nummerierte o-Simplex $v(S)=\left(v\left(x_{0}\right), v\left(x_{1}\right), \ldots, v\left(x_{d}\right)\right)$. Jenes o-Simplex $S$, das zum lexikographisch kleinsten nummerierten o-Simplex $v(S)$ führt, nennen wir ein Referenzsimplex von $P$ und bezeichnen es mit $\operatorname{Ref}_{P}$. Für jede Minimums-Nummerierung von $P$ lässt sich so ein Referenzsimplex $\operatorname{Re} f_{P}$ angeben. Da aber alle Referenzsimplexe $R e f_{P}$ durch Automorphismen auseinander hervorgehen, sind sie isometrisch und aufgrund der Chiralität von $P$ gleich orientiert, was folgende Definition ermöglicht:

Definition 13.1. Zwei chirale $d$-dimensionale Polytope $P$ und $Q$ sind gleich orientiert, wenn zwei Referenzsimplexe $\operatorname{Re} f_{P}$ und $R e f_{Q}$ gleich orientiert sind, sonst verschieden orientiert.

\section{Bemerkungen}

(1) Eine Orientierung beinhaltet immer eine Willkür. Bei unserem Verfahren werden, ausgehend von der Darstellung eines Polytops mit einer gewählten Reihenfolge der Metrikrelationen, zwei Minimierungen vorgenommen: Eine erste bei der Wahl der Nummerierungsklasse und eine zweite bei der Wahl der Referenztupel bezüglich dieser Klasse.

(2) Nach E. Ruch [10] gibt es spezielle Molekülklassen mit der Möglichkeit einer Orientierung von ,physikalischer Relevanz', insofern als bei stetiger Veränderung physikalischer Parameter auf dem Weg von einer Chiralitätsklasse zur andern immer ein achiraler Zustand durchlaufen werden muss. In entsprechender Weise lässt sich mit Hilfe von Stetigkeit bei speziellen Polytopklassen eine Orientierung von ,geometrischer Relevanz‘ definieren.

Bei koordinaten-abhängigen chiralen $d$-dimensionalen Polytopen ist die analytische Umsetzung der Definition 13.1 mittels eines Orientierungssinns naheliegend: Ist $\operatorname{Re}_{P}$ ein $\operatorname{Re}$ ferenzsimplex eines solchen Polytops $P$, so heisst $P$ positiv orientiert falls $\operatorname{det}\left(\operatorname{Re} f_{P}\right)>0$ und negativ orientiert falls $\operatorname{det}\left(\operatorname{Re} f_{P}\right)<0$. Bei den Wahrnehmungsräumen der Dimensionen $d=2$ und $d=3$ lässt sich für die Referenzdreiecke bzw. -tetraeder in bekannter 
Weise ein anschaulicher Orientierungssinn verwenden, nämlich rechts orientiert (,Gegenuhrzeigersinn ' bzw. ,Rechte-Hand-Regel' ) und links orientiert. Setzt man bei der Determinantendefinition wie üblich ein rechts orientiertes Koordinatensystem voraus, so liefern positiv und rechts orientiert und somit negativ und links orientiert dieselben Chiralitätsklassen von Polygonen bzw. Polyedern. Erwähnt sei noch, dass auch ohne Verwendung eines Orientierungssinns entschieden werden kann, ob zwei chirale $d$-dimensionale Polytope $P$ und $Q$ gleich oder verschieden orientiert sind, vorausgesetzt man kennt die gegenseitige Lage zweier Referenzsimplexe $R e f_{P}$ und $R e f_{Q}$. Dies geschieht dann anhand des Vorzeichens von $\operatorname{det}\left(\operatorname{Re} f_{P}, \operatorname{Re} f_{Q}\right)$ gemäss Satz 11.1.

Von den bisher betrachteten Polytopen sind drei chiral, nämlich das Parallelogramm $P a$ $(d=2)$, die Bipyramide $B p(d=3)$ und das Polytop Pt $(d=4)$. In diesen drei Beispielen ergibt sich als Orientierungssinn:

$P a$ : Zwei Minimums-Nummerierungen $v_{1}$ und $v_{2}$ (10.2), wir wählen $v_{1}$ : $R e f_{P a}=(b, c, d)$, also $P a$ rechts (vgl. Fig. 9) bzw. positiv orientiert;

$B p$ : $\quad$ Nur eine Minimums-Nummerierung $v(9.1)$ : $R e f_{B p}=(d, c, e, a)$, also $B p$ links (vgl. Fig. 8) bzw. negativ orientiert;

$P t$ : $\quad$ Drei Minimums-Nummerierungen $v_{1}, v_{2}$ und $v_{3}$, wir wählen $v_{2}$ (12.1): $(e, g, d, a, f)$ und $(e, g, d, a, c)$ keine Referenzsimplexe (da entartet), $R e f_{P t}=(e, g, d, a, b) \operatorname{mit} \operatorname{det}\left(\operatorname{Re} f_{P t}\right)=8$, also $P t$ positiv orientiert.

\section{Polygone und Polyeder}

Die speziellen Polytope (wir meinen nach wie vor konvexe), die Polygone und Polyeder, lassen eine Reduktion der Eckenpaare bei den Darstellungen zu, ohne dass sich die mit Hilfe des Minimierungsalgorithmus generierte Automorphismengruppe ändert. Bei einem $n$-eckigen Polygon oder Polyeder kann nämlich die quadratische Anzahl von Eckenpaaren $n(n-1)$ theoretisch auf eine lineare Anzahl gesenkt werden. Bei den folgenden Ausführungen bleibt vieles nur angedeutet.

Zunächst betrachten wir die $n$-eckigen Polygone. Ein solches Polygon ist bis auf Isometrie bereits eindeutig festgelegt durch die ,Kantenmetrik“ (Abstände zwischen je zwei auf dem Rand aufeinanderfolgenden Ecken) und die ,Erstdiagonalmetrik‘ (Abstände zwischen je einer Ecke und der auf dem Rand übernächsten). Der Beweis dieser Aussage erfolgt induktiv und sieht im Wesentlichen so aus: Beginnend mit einem Randdreieck (bis auf Isometrie bestimmtes Dreieck bestehend aus zwei aufeinanderfolgenden Kanten und einer Erstdiagonalen) ist jeweils pro Induktionsschritt, unter Berücksichtigung der Konvexität, das nächste Randdreieck anzufügen. Bei der Darstellung müssen so für $n \geq 5$ nur $4 n$ Eckenpaare (pro Abstand wie üblich beide Paare) angegeben werden. Es ist naheliegend, die Kanten- und Erstdiagonalmetrik mit gesonderten Metrikrelationen zu erfassen. Im Spezialfall eines regulären Polygons genügt die Angabe der Kantenmetrik, und die Darstellung hat dann eine einzige Metrikrelation mit $2 n$ Eckenpaaren.

Bei Polygonen kann man sich beim Chiralitäts-Test auf den Automorphismen-Test beschränken, denn als Symmetrie- und damit Automorphismengruppen kommen nur die 
zyklische Gruppe $C_{m}(m \geq 1)$ bei chiralen und die Diedergruppe $D_{m}(m \geq 1)$ bei achiralen Polygonen in Frage. Einzige Ausnahme bildet die Gruppenordnung 2, wo $C_{2}$ und $D_{1}$ isomorph sind und deshalb ein Simplex-Test notwendig sein kann, wie anhand der Polygone $T r$ und $P a$ dargelegt wurde (siehe Abschnitt 10).

Was die $n$-eckigen Polyeder betrifft, so beruht die Reduktion der Eckenpaare auf dem sogenannten ,Starrheitssatz von Cauchy ' [3] aus dem Jahre 1813. Anschaulich formuliert besagt dieser Satz Folgendes: Denkt man sich bei einem (immer noch konvexen) Polyeder die Randpolygone aus Metallplatten gefertigt und die Kanten mit Scharnieren versehen, so ist das Polyeder starr. Oder präziser formuliert: Ein Polyeder ist durch die ,Randmetrik $^{\star}$ - das, was man von aussen sieht - bis auf Isometrie eindeutig festgelegt (bei der Bipyramide $B p$ in Fig. 8 wäre somit die Angabe der Länge der im Innern verlaufenden Eckenverbindung $a c$ überflüssig). Bei der Randmetrik genügt es, nebst der Kantenmetrik die Erstdiagonalmetrik aller Randpolygone zu erfassen, auch hier mit gesonderten Metrikrelationen. Mit Hilfe des Eulerschen Polyedersatzes lässt sich beweisen, dass so bei der Darstellung stets weniger als 10n Eckenpaare anzugeben sind. In Spezialfällen, etwa bei einem regulären oder halbregulären Polyeder, ist sogar eine Beschränkung auf die Kantenmetrik möglich.

Im Unterschied zu Polygonen kann bei Polyedern für den Chiralitäts-Test ein SimplexTest erforderlich sein. Arbeitet man ,koordinaten-frei', so verlangt der Simplex-Test im Allgemeinen die Kenntnis von Abständen zwischen Ecken, deren Verbindungsstrecken durch das Innere des Polyeders laufen; die Randmetrik ist nicht ausreichend.

Wie aber bestimmt man die Eckenpaare, die bei der reduzierten Darstellung von Polygonen und Polyedern zu verwenden sind? Im Normalfall erfordert das Aussortieren dieser Eckenpaare einen zusätzlichen Algorithmus, was den Nutzen der Reduktion in Frage stellt. Es sei noch hinzugefügt, dass durch die Definition 13.1 eine andere Orientierung von chiralen Polygonen bzw. Polyedern festgelegt wird, wenn man anstelle der vollen eine reduzierte Darstellung benützt.

\section{Schlussbemerkungen}

Es gibt eine Vielzahl weiterer interessanter Themen im Zusammenhang mit Chiralität. So hat beispielsweise die Grenze zwischen lebender und nicht-lebender Natur etwas mit Chiralität zu tun. Bei den Molekülen des Lebens, etwa bei den Aminosäuren als Bausteine von Proteinen, kommt gewöhnlich nur eines der beiden Enantiomeren vor. Für dieses Phänomen der sogenannten Homochiralität gibt es verschiedene Erklärungsversuche, die unter anderem auch auf statistische Probleme führen [11]. Mathematisch reizvoll ist auch die Frage nach einem Chiralitätsmass, einem Mass für Abweichung von Achiralität. Ein interessanter Beitrag dazu stammt von der Arbeitsgruppe um den Chemiker K. Mislow (Dr. h.c. 2004 der Universität Zürich), die mit dem geometrischen Konzept der HausdorffMetrik arbeitet. In [2] werden in einer Übersicht Beiträge zu diesem Thema diskutiert.

Kant hat unseres Wissens als erster das Phänomen der Chiralität mit Hilfe von enantiomeren Händen sorgfältig beschrieben. Ganz abgesehen von der grossen praktischen Bedeutung, etwa in der modernen Chemie, ist Chiralität ein reizvolles Thema im Grenzbereich von Philosophie, Mathematik und Naturwissenschaft. 


\section{Literatur}

[1] Boissonnat, J.-D.; Yvinec, M.: Algorithmic Geometry. Cambridge Univ. Press, Cambridge 1998.

[2] Buda, A.B.; Auf der Heyde, T.; Mislow, K.: Quantifizierung der Chiralität. Angew. Chem. 104 (1992), 1012-1031.

[3] Cauchy, A.L.: Sur les Polygones et Polyèdres, (second mémoire). J. de l'Ecole Polytechnique XVIe Cahier, Tome IX (1813), 87-98. In: Oeuvres Complètes d'Augustin Cauchy. IIe Série, Vol. 1, ed. Académie des Sciences, Paris 1905, 26-38.

[4] Floersheim, P.; Wirth, K.; Huber, M.K.; Pazis, D.; Siegerist, F.; Haegi, H.R.; Dreiding, A.S.: From Mobile Molecules to their Symmetry Groups: A Computer-Implemented Method. Stud. Phys. Theor. Chem. 23 (1983), 59-80.

[5] Grünbaum, B.: Convex Polytopes. 2nd edition, Springer, New York 2003.

[6] Kant, I.: Königsbergische Frag- und Anzeigungs-Nachrichten. M. Hoffmann (ed.) 1768 (Faksimile von 1920).

[7] Klein, K.: Algorithmische Geometrie. Addison Wesley, Bonn 1997.

[8] Prelog, V.: Chirality in Chemistry. Science 193 (1976), 17-24, oder Internet: http://nobelprize.org/chemistry/laureates/1975/prelog-lecture.html

[9] Reidemeister, K.: Raum und Zahl. Springer, Berlin-Göttingen-Heidelberg 1957.

[10] Ruch, E.: Homochiralität als Klassifizierungsprinzip von Molekülen spezieller Molekülklassen. Theoret. chim. Acta (Berl.) 11 (1968), 183-192.5

[11] Siegel, J.S.: Homochiral Imperative of Molecular Evolution. Chirality 10 (1998), 24-27.6

[12] Wirth, K.; Huber, M.K.: Numbering of Finite Relational Systems. Match 12 (1981), 3-14.

[13] Wirth, K.: Coding of Relational Descriptions of Molecular Structures. J. Chem. Inf. Comput. Sci. 26 (1986), 242-249.

[14] Ziegler, G.M.: Lectures on Polytopes. Springer-Verlag, New York 1995 (rev. ed. 1998).

Karl Wirth, André S. Dreiding

Organisch-Chemisches Institut

Universität Zürich

Winterthurerstrasse 190

CH-8057 Zürich, Switzerland

e-mail:wirthk@gmx.ch, asd@oci.unizh.ch

\footnotetext{
${ }^{5}$ Der Begriff ,Homochiralität' ${ }^{\star}$ hat hier eine etwas andere Bedeutung als in [11]

${ }^{6}$ Siehe auch im Internet: Stichwort ,Homochiralität' oder ,homochirality'.
} 